\title{
Brain Tumor Detection using Marpholical Segmentation Techniques
}

\author{
K.Shankar ${ }^{1}$, A.Parimala ${ }^{2}$, M.V.V.S.T. Chaitanya ${ }^{3}$, T.Supriya ${ }^{4}$ \\ Associate Professor, Dept of C.S.E, Nadimpalli Satyanarayana Raju Institute of Technology, Visakhapatnam, India ${ }^{1}$ \\ B.Tech., Student, Dept of C.S.E, Nadimpalli Satyanarayana Raju Institute of Technology, Visakhapatnam, India ${ }^{2,3,4}$
}

\begin{abstract}
In medical image processing there are many challenging tasks and performing the segmentation on a brain image is more difficult to understand and extract the tumor form it and classifying the detection rate in MRI images so image segmentation and identification of tumor is one of the best methods for identifying the tumor in image so here in this paper we implement the watershed algorithm and contrast enhancement technique in segmenting a brain image which gives us more accuracy.
\end{abstract}

Keywords: Segmentation, Brain Tumor. Watershed,MRI

\section{INTRODUCTION}

Automated classification and detection of tumors indifferent medical images is motivated by the necessity of high accuracy when dealing with a human life. Also, the computer assistance is demanded in medical institutions due to the fact that it could improve the results of humans in such a domain where the false negative cases must be at a very low rate. It has been proven that double reading of medical images could lead to better tumor detection. Butte cost implied in double reading is very high, that's why good software to assist humans in medical institutions is of great interest nowadays. Conventional methods of monitoring and diagnosing the diseases rely on detecting the presence of particular features by a human observer. Due to large number of patients in intensive care units and the need for continuous observation of such conditions, several techniques for automated diagnostic systems have been developed in recent years to attempt to solve this problem. Such techniques work by transforming the mostly qualitative diagnostic criteria into a more objective quantitative feature classification problem In this project the automated classification of brain magnetic resonance images by using some prior knowledge like pixel intensity and some anatomical features is proposed. Currently there are no methods widely accepted therefore automatic and reliable methods for tumor detection are of great need and interest. The application of PNN in the classification of data for MR images problems are not fully utilized yet. These included the clustering and classification techniques especially for MR images problems with huge scale of data and consuming times and energy if done manually. Thus, fully understanding the recognition, classification or clustering techniques is essential to the developments of Neural Network systems particularly in medicine problems.

Segmentation of brain tissues in gray matter, white matter and tumor on medical images is not only of high interest in serial treatment monitoring of "disease burden" in oncologic imaging, but also gaining popularity with the advance of image guided surgical approaches. Outlining the brain tumor contour is a major step in planning spatially localized radiotherapy (e.g., Cyber knife, IMRT ) which is usually done manually on contrast enhanced T1-weighted magnetic resonance images (MRI) in current clinical practice. On T1 MR Images acquired after administration of a contrast agent (gadolinium), blood vessels and parts of the tumor, where the contrast can pass the blood-brain barrier are observed as hyper intense areas. There are various attempts for brain tumor segmentation in the literature which use a single modality, combine multi modalities and use priors obtained from population atlases.

\section{RELATED WORK}

In this paper, Gopal et al [1] proposed a smart system it is designed to diagnose brain tumor through MRI using image processing clustering algorithms such as fluffy $\mathrm{C}$ Means along with intelligent optimization tools, such as Genetic Algorithm (GA), and Particle Swarm Optimization (PSO). The average results classification error of GA is $0.078 \%$. The average accuracy GA is $89.6 \%$. PSO gives best classification accuracy and average error rate. In this the Average 


\section{International Journal of Advanced Research in Computer and Communication Engineering}

Vol. 8, Issue 2, February 2019

classification error of PSO is $0.059 \%$ and the accuracy is $92.8 \%$ and tumor detection is $98.87 \%$. Therefore, we saw that average classification error is reduced when the number of sample is increased. This report has provided substantial evidence that for brain tumor segmentation of PSO algorithm performed well.In this paper, Badran et al [2], proposed an innovative system which can be used as a second decision for the surgeons and were based on adaptive thresholding. It determines whether an input MRI brain image represents a healthy brain or tumor brain as percentage.it defines the tumor type; malignant or benign tumor.

In this paper, Viji et al [3], uses watershed segmentation technique at the desired parameters, which results the watershed segmentation is the best method to segment a tumor in MATLAB, provided.In this paper, Aka et al [4], Segmentation and Detection ofbrain tumor is done using MR images. This method improved the MR image and segments the Tumor Using global thresholding. This method performs well in enhancing, segmenting and extracting the Brain Tumor from MRI images.

In this paper, Dubey et al [5], a relationship of the different semi-automated methods, modified gradient magnitude region growing technique (MGRRGT), level set and a marker controllex watershed method is undertaken here for evaluating their relative performance in the segmentation of tumor. The results show that area measurements obtained using MGMRGT method is in good agreement with manually segmented data. In this paper, Krishnan et al [6], say about take out brain tumor from MRI brain images using image processing, segmentation, morphological operations and subtraction. Some of the morphological operators that can change the structuring elements of an image as stated to their use are open and close has helped in extracting the tumor from the MRI brain image. The exact shape of the tumor in that MRI image and finally detection of brain tumor in MRI image is achieved. In this paper, Ghanavati et al [7], it causes to an automatic tumor detection algorithm using multi-modal MRI. The results show 100\% detection rate in all our test sets including simulated and patient data with an average accuracy of $90 \%$. In this paper, Bhattacharjee et al [8], a new technique for brain tumor detection from diseased MR images is developed in it. This would enhance the efficiency of the detection and would stretch it to further disease classification. In this paper, Yao-Tien Chen [9], a new method proposes an approach integrating 3D Bayesian level set methodwith volume rendering for brain tumor and tissue segmentation and rendering.

\section{METHODOLOGY}

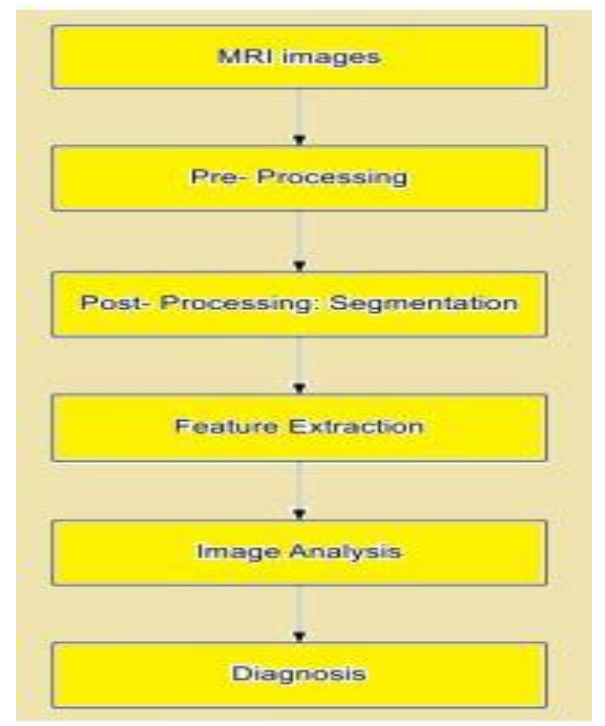

Fig 1 proposed Method

\section{1) Median Filtering for Noise Removal}

Median filter is a non-linear filtering technique used for noise removal.[5] Median filtering is used to remove salt and pepper noise from the converted gray scale image. It replaces the value of the center pixel with the median of the intensity values in the neighborhood of that pixel. Median filters are particularly effective in the presence of impulse noise. Impulse noise is also called as salt and pepper noise because of its appearance as white and black dots covered on image. The median filter is used for remove salt and pepper noise from MRI images is shown in figure 2 
Vol. 8, Issue 2, February 2019

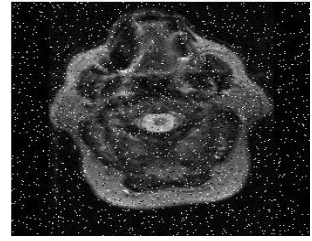

Fig. 2 (a) Salt and pepper Noise

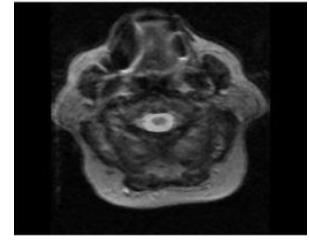

(b) Median filter apply

\section{2)Image Enhancement}

Poor contrast is one of the defects found in acquired image. The effect of that defect has great impact on the contrast of image. When contrast is poor the contrast enhancement method plays an important role. In this casePoor contrast is one of the defects found in acquired image. The effect of that defect has great impact on the contrast of image. When contrast is poor the contrast enhancement method plays an important role. In this case the gray level of each pixel is scaled to improve the contrast. Contrast enhancements improve the visualization of the MRI images. [8] contrast enhancement techniqe

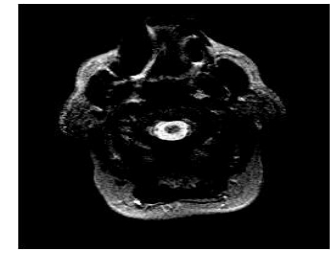

Fig.3 (a) Reduced Contrast

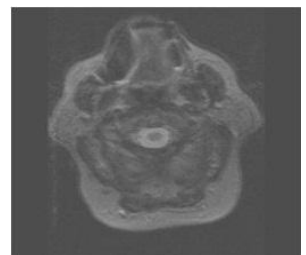

Fig. 3 (b) Increase contrast

\section{3) Edge Detection}

Edge detection is an image processing technique for finding the boundaries of objects within images. It works by detecting discontinuities in brightness. Edge detection is used for image segmentation and data extraction in areas such as image processing, computer vision, and machine vision. Common edge detection algorithms include methods like Sobel, Canny, Prewitt, Log, and Zero cross. Edge detection methods are used for finding object boundaries from MRI images and the results are shown in figure 4

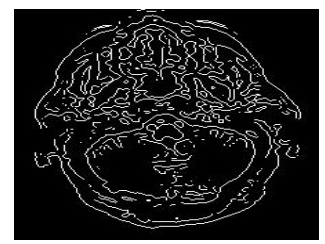

Fig. 4 (a) sobel Filtering

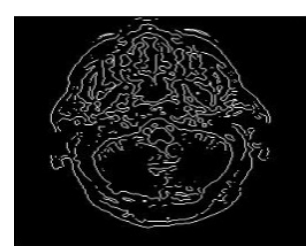

Fig. 4 (b) winner filtering

\section{4) Threshold}

Image threshold is a simple, effective, way of partitioning an image into a foreground and background. This image analysis technique is a type of image segmentation that isolates objects by converting gray scale images into binary images. Image threshold is most effective in images with high levels of contrast. threshold technique is apply on input MRI image by changing the threshold value and the result shown in figure 5.

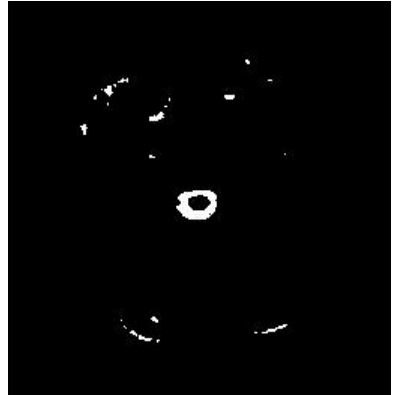

Fig. 5. Threshold applied image 


\section{International Journal of Advanced Research in Computer and Communication Engineering}

Vol. 8, Issue 2, February 2019

\section{5) Morphological Operation}

Morphological operation used as an image processing tools for sharpening the regions. [2] Morphological image processing is a collection of non-linear operations related to the shape or morphology of features in an image. The erosion and dilation methods are use for morphological operation.[16]

\section{6) Segmentation}

Image segmentation is the process of partitioning a digital image into multiple segments. Image Segmentation is typically used to locate objects and boundaries in image, image segmentation is the process of assigning a label to every pixel in an image such that pixels with the same label share certain visual characteristics [14]. The different segmentation techniques are shown in table 2 [2]- [15].

\section{EXPERIMENTAL/SETUP AND RESULTS}

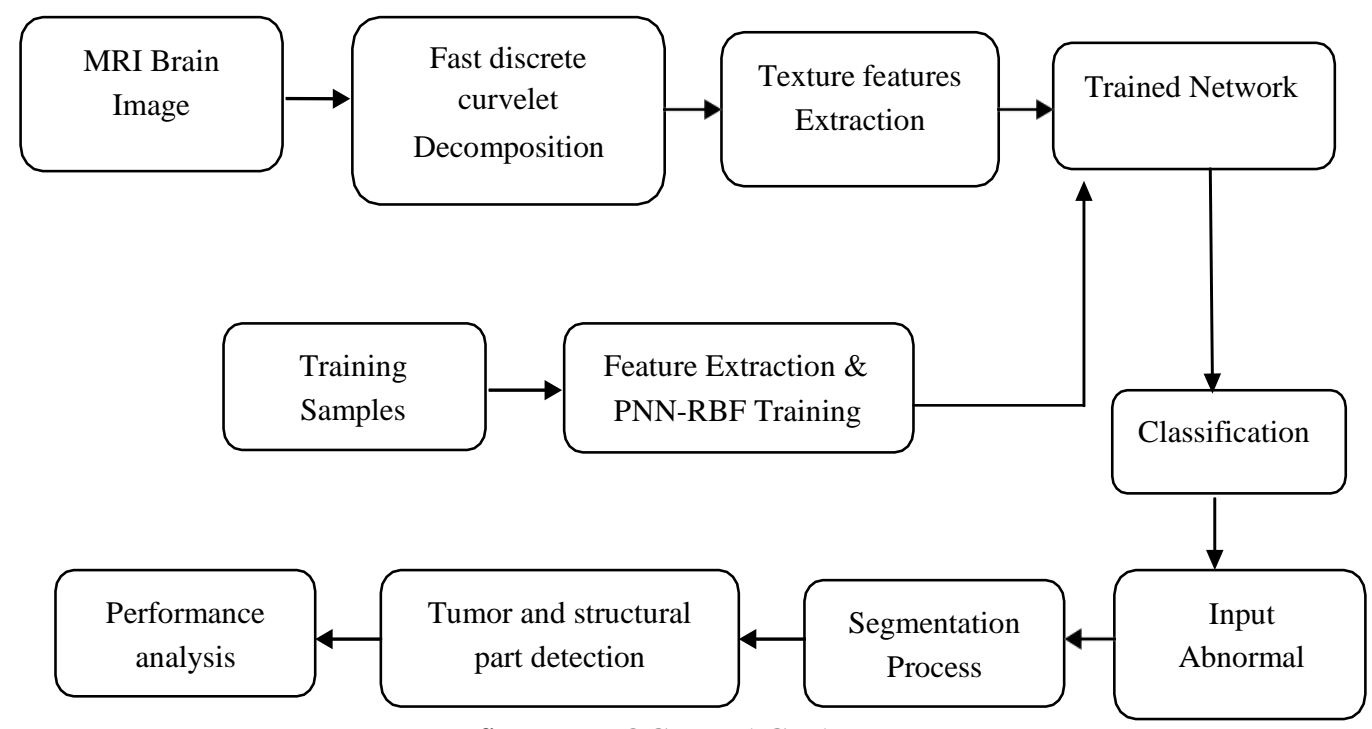

fig 6: BLOCK DIAGRAM

In the last two or three years, however, Curvelets have actually been redesigned in a effort to make them easier to use and understand. As a result, the new construction is considerably simpler and totally transparent. What is interesting here is that the new mathematical architecture suggests innovative algorithmic strategies, and provides the opportunity to improve upon earlier implementations. The two new fast discrete curvelet transforms (FDCTs) which are simpler, faster, and less redundant than existing proposals:

$>\quad$ Curvelets via USFFT .

$>\quad$ Curvelets via Wrapping.

The block size can be changed at each scale level. The wrapping construction is shown is taken to be a Cartesian array and ^ $f[n 1, n 2]$ denotes its 2-D discrete Fourier transform, then the architecture of the FDCT via wrapping is as follows.

* Apply the 2-D FFT and obtain Fourier samples,

$$
\hat{f}\left[n_{1}, n_{2}\right], \quad-\frac{n}{2} \leq n_{1}, \quad n_{2}<\frac{n}{2} .
$$

* For each scale $j$ and angle $l$, form the product

$$
\hat{U}_{j, l}\left[n_{1}, n_{2}\right] \hat{f}\left[n_{1}, n_{2}\right]
$$

* Wrap this product around the origin and obtain

* Apply the inverse 2-D FFT to each $\sim f j, l$, hence collecting the discrete coefficient

$$
\tilde{f}_{j, l}\left[n_{1}, n_{2}\right]=W\left(\tilde{U}_{j, l} \hat{f}\right)\left[n_{1}, n_{2}\right]
$$




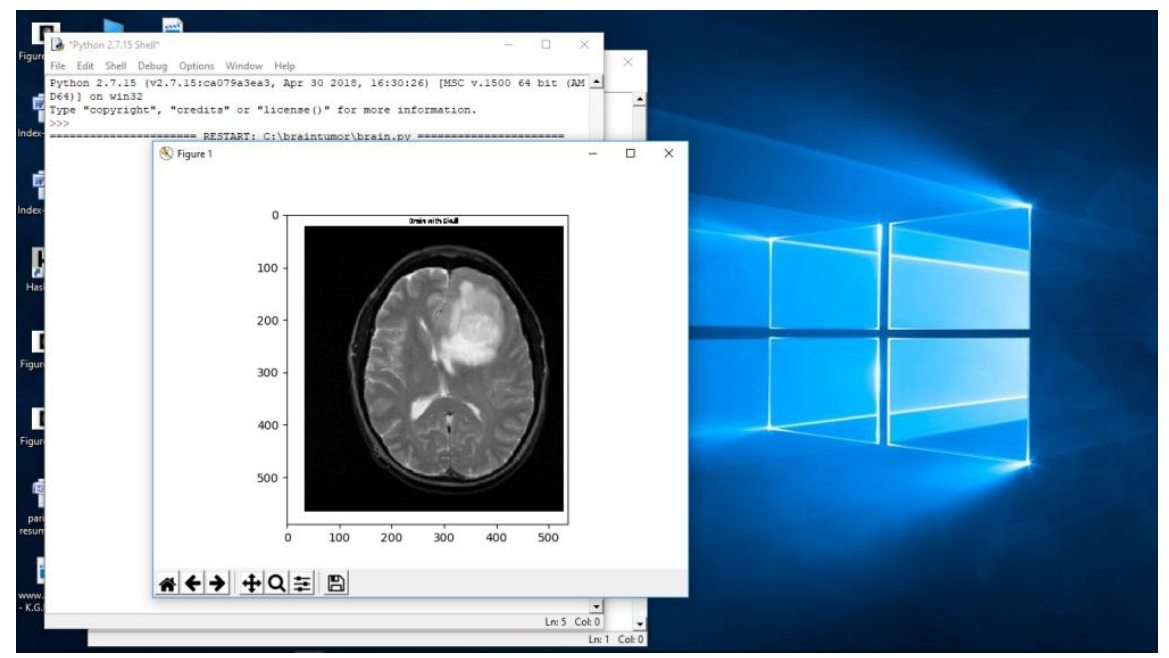

Fig 7 input tumor image

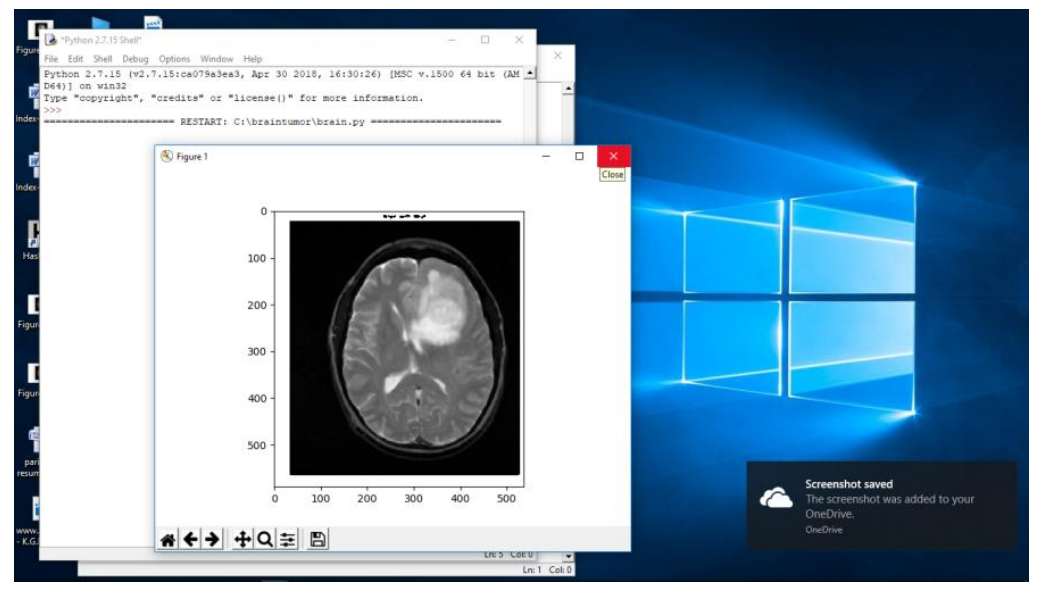

Fig 8 applying the filters and perform edged detection

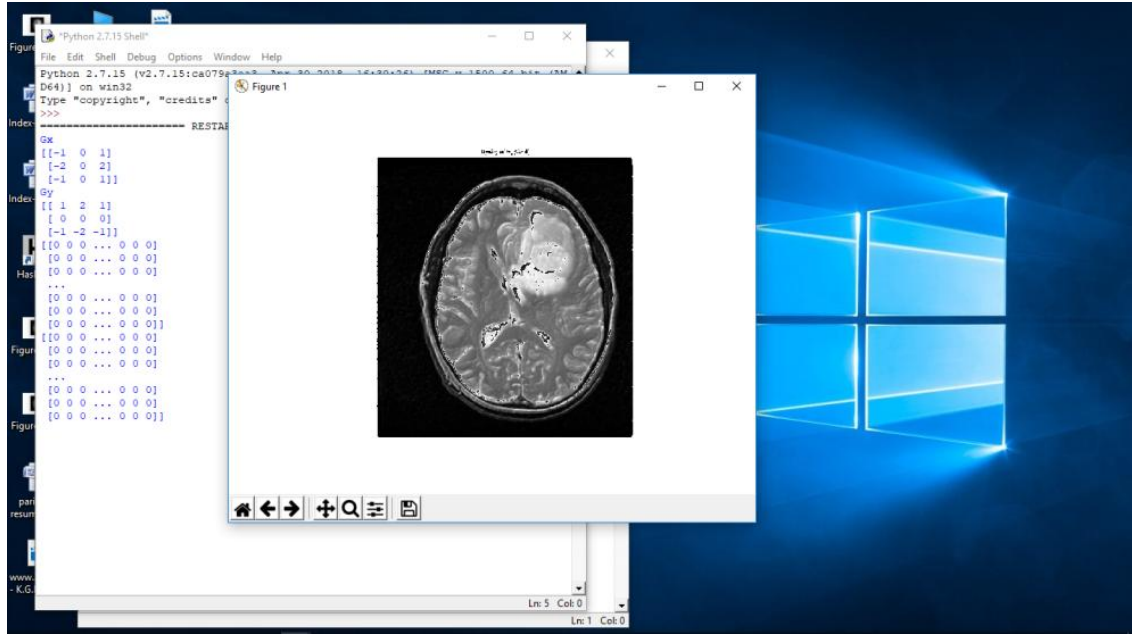

fig 9 getting the segment area if tumor 
Vol. 8, Issue 2, February 2019

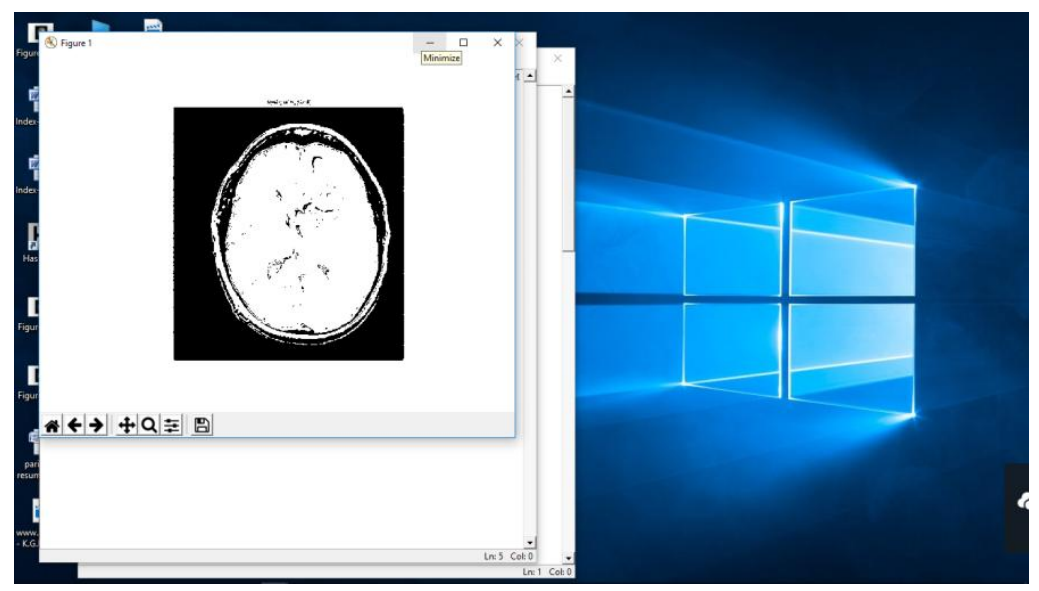

Fig 10 : background and foreground substraction

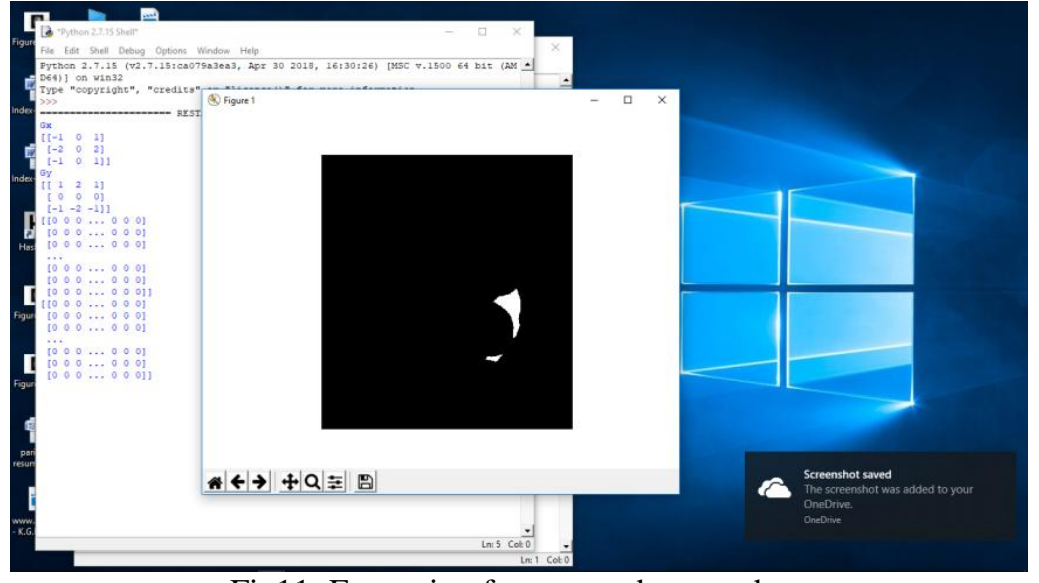

Fig11 :Extracting fore ground tumor data

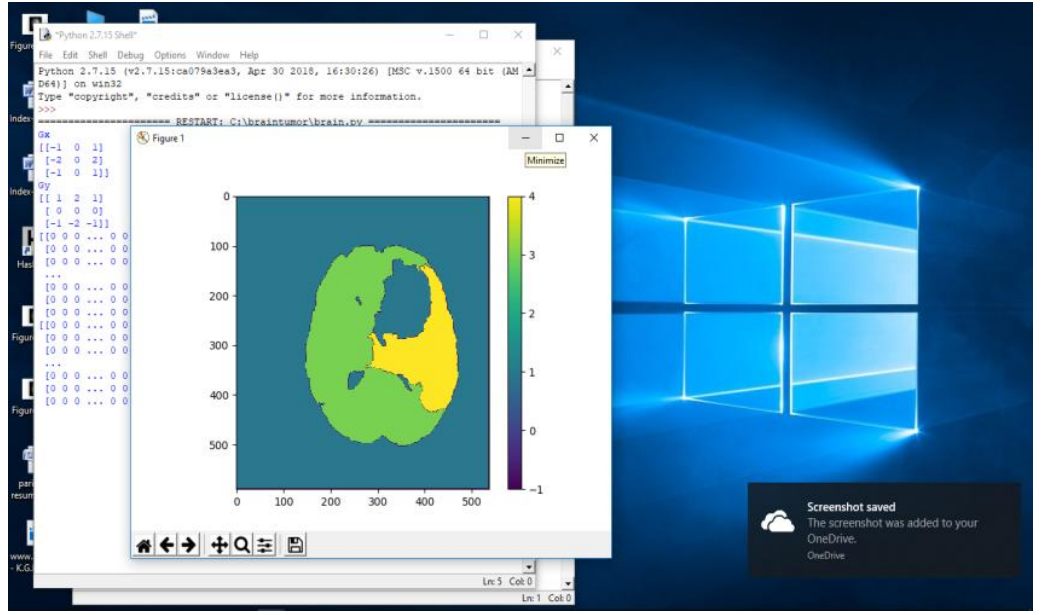

Fig 11 : Applying watershed algorithm to identify for ground and background 


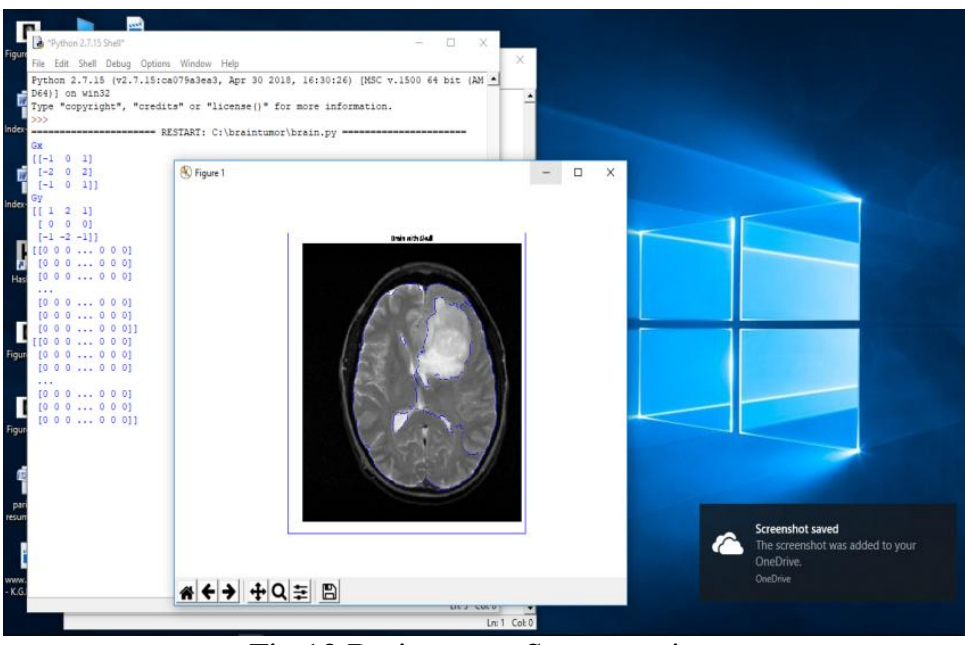

Fig 12 Brain tumor Segmentation

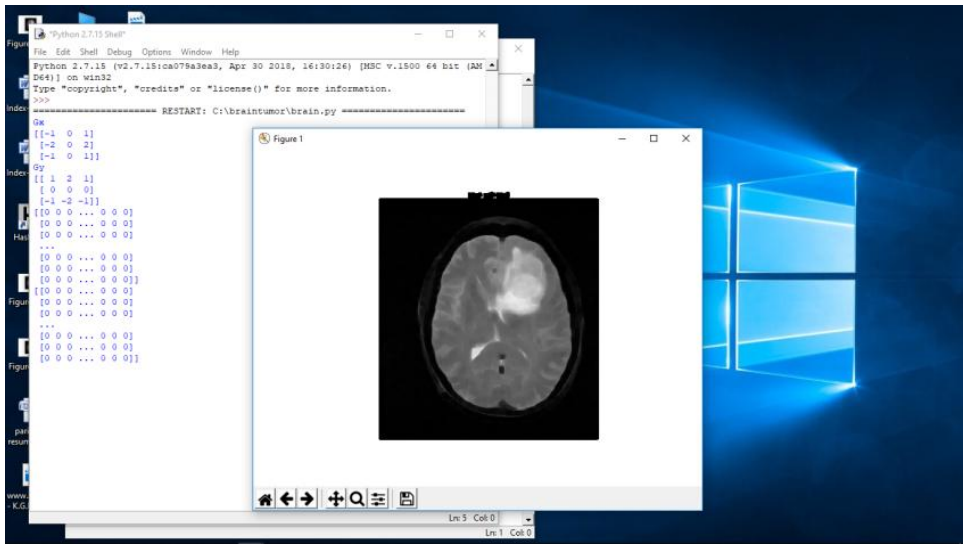

Fig 13 Identified Brain Tumor Region

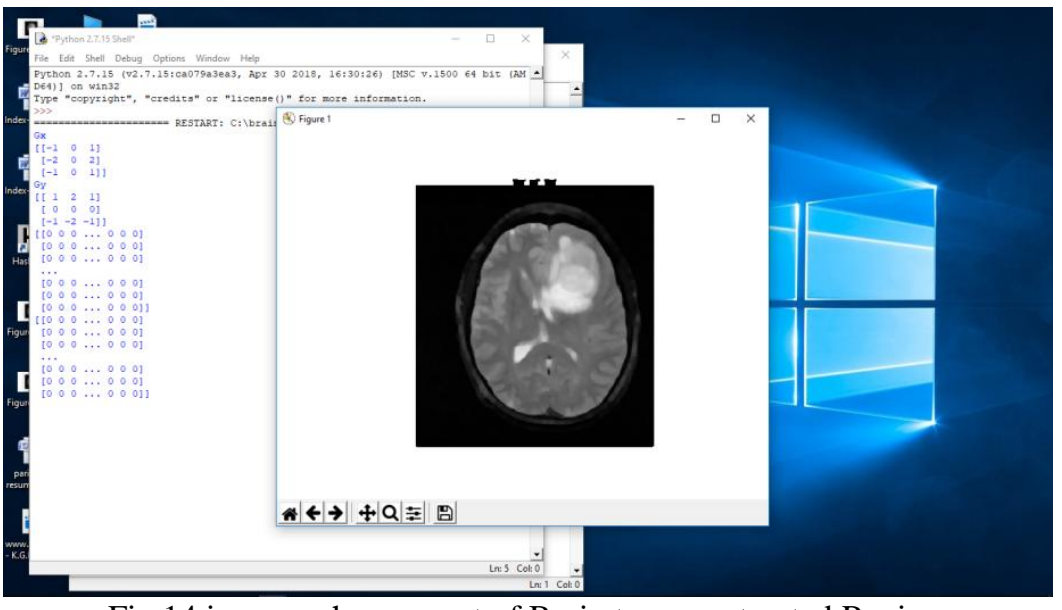

Fig 14 image enhancement of Brain tumor extracted Region 


\section{International Journal of Advanced Research in Computer and Communication Engineering}

Vol. 8, Issue 2, February 2019

\section{CONCLUSION AND FUTURE SCOPE}

MRI images are best suitable for brain tumor detection. In this study Digital Image Processing Techniques are important for brain tumor detection by MRI images. The preprocessing techniques include different methods like Filtering, Contrast enhancement, Edge detection is used for image smoothing. The preprocessed images are used for post processing operations like; threshold, histogram, segmentation and morphological, which is used to enhance the images.

\section{REFERENCES}

[1] N. Nandha Gopal, "Dr. Karnan,Diagnose Brain Tumor Through MRI using Image Processing Algorithm such as Fuzzy C Means Along with Intelligent Optimization Techniques", journal of IEEE 2010.

[2] Ehab F. Badran, Esraa Galal Mahmoud, and Nadder Hamdy, "An Algorithm for Detecting Brain tumors in MRI Images”, journal of IEEE 2010.

[3] K.S Angel Viji, Dr. J.Jayakumari, "Automatic Detection of Brain Tumor based on MRI Using CAD system and watershed segmentation", Proceeding International conference on Signal processing, communication, and Networking technology, 2011.

[4] M. Usma aka Ana Usma, "Computer Aided Brain Tumor Detection and Segmentation", Journal of IEEE, 2011.

[5] R.B.Dubey, M.Hanmandlu, Sr. Member, Shantaram Vasikarla, "Evaluation of three Methods for MRI Brain Tumor Segmentation", Eighth International Conference on Information Technology, 2011.

[6] Natranjan.p, Krishnan.N, Natasha Sandeep Kenkre, Shraiya Nancy, Bhuvanesh Pratap singh, "Tumor Detection using threshold operation in MRI Brain Image", IEEE International Conference on Computational Intelligence and Computing Research, 2012.

[7] Sahar Ghanavati, Junning, Ting Liu, Paul S. Babyn, Wendy Doda, George Lampropoulous, "Automatic Brain Tumor Detection in MR images", journal of IEEE 2012.

[8] Rupsa Bhattacharjee, Dr. Monisha Chakarborty, "Brain Tumor Detection from MR images: Image Processing, Slicing and PCA based Reconstruction”, Proceeding International conference on Emerging Applications of Information Technology, 2012.

[9] Yao-Tien Chen, "Brain Tumor Detection using 3-D Bayesian Level set Method with Volume Rendering", Proceeding International conference on Wavelet Analysis and Pattern Recognition, Xian,2012.

[10] M. Monica Subashinim, Sarat Kumar Sahoo, "Brain Tumor Detection using PCNN and BPN", Third International conference on Sustainable energy and Intelligence system, 2012. 